\title{
Estudio del comportamiento del acero 2.25Cr-1Mo frente a los fenómenos de oxidación cíclica e isoterma
}

\author{
Manuel Proy ${ }^{\mathrm{a}, \varpi}$, María Victoria Utrilla ${ }^{\mathrm{a}}$, Enrique Otero $^{\mathrm{a}}$ \\ aDpto. de Ciencia e Ingeniería de Materiales, Universidad Rey Juan Carlos, C/ Tulipán s/n 28933, Móstoles, Madrid, España \\ $\bowtie$ Autor para la correspondencia: manuproy@gmail.com
}

RESUMEN: La resistencia del acero aleado con cromo y molibdeno $2.25 \mathrm{Cr}-1 \mathrm{Mo}$ frente a la oxidación isotérmica y cíclica, fue evaluada a $550{ }^{\circ} \mathrm{C}$ y $650{ }^{\circ} \mathrm{C}$ durante 360 horas en aire. Los ciclos consistieron en el mantenimiento durante 90 minutos dentro del horno a la temperatura de ensayo y un periodo de enfriamiento forzado hasta $50{ }^{\circ} \mathrm{C}$ mediante la aplicación de un flujo de aire. Se realizó un estudio termogravimétrico para calcular las cinéticas de oxidación. Para conocer el mecanismo de degradación, se emplearon diversas técnicas de caracterización microestructural, como Difracción de Rayos (DRX), Microscopía Óptica (MO) y Electrónica de Barrido (MEB). El ciclado térmico cambia el mecanismo de degradación, debido a la propagación de grietas en la película de óxido formada, que favorece el acceso del agente agresivo al sustrato.

PALABRAS CLAVE: Acero empleado en calderas; Ciclado térmico; Cinéticas de corrosión; Corrosión a alta temperatura

Citation / Cómo citar este artículo: Proy, M., Utrilla, M.V., Otero, E. (2014) "Estudio del comportamiento del acero 2.25Cr-1Mo frente a los fenómenos de oxidación cíclica e isoterma". Rev. Metal. 50(1): e003. doi: http://dx.doi org/10.3989/revmetalm.003

SUMMARY: Cyclic and isothermal oxidation behavior of $2.25 \mathrm{Cr}-1 \mathrm{Mo}$ steel. Cyclic and isothermal oxidation of chromium-molybdenum steel $2.25 \mathrm{Cr}-1 \mathrm{Mo}$ were analyzed at $550^{\circ} \mathrm{C}$ and $650^{\circ} \mathrm{C}$ during 360 hours in air atmospheres. The cycles were performed with two stages; one of heating in furnace during 90 minutes and then the sample were cooled to $50{ }^{\circ} \mathrm{C}$ by air flow. Thermogravimetric analyses were performed to obtain high temperature corrosion kinetics. Several characterization techniques have been used to identify the corrosion mechanism, as X-Ray Diffraction (XRD), Optical Microscopy (OM) and Scanning Electron Microscopy (SEM). Thermal cycling tests can changes the corrosion mechanism, due cracks propagation in oxide scale, that witch can favors the access of oxidant agent to the substrate.

KEYWORDS: Boiler steel; High temperature corrosion; Kinetic law; Thermal cycling

Copyright: $\odot$ CSIC. This is an open-access article distributed under the terms of the Creative Commons AttributionNon Commercial (by-nc) Spain 3.0 License.

\section{INTRODUCCIÓN}

Uno de los principales objetivos de los últimos años es disminuir las emisiones contaminantes producidas en las centrales térmicas convencionales de combustibles fósiles. Por tanto, es necesario desarrollar nuevas tecnologías que permitan aumentar el rendimiento energético (Viswanathan y Bakker, 2001). El principal factor que limita la eficiencia del proceso completo, es la diferencia de temperatura 
entre el foco caliente y frío del ciclo, es decir, desarrollando tecnologías de materiales que permitan dicho incremento de temperatura, es posible incrementar la eficiencia, disminuir costes y limitar las emisiones contaminantes al exterior (Sarver et al., 2006). Los materiales expuestos en condiciones de alta temperatura, deben ser capaces de operar correctamente, manteniendo su integridad estructural frente a fenómenos como la fluencia, la fatiga y la corrosión (Henry et al., 2005).

Asimismo, durante la operación en servicio, las centrales pueden estar sometidas a ciclos de arranque y parada, o bien a fluctuaciones térmicas que pueden afectar gravemente al comportamiento del material (Young, 2008). La acción combinada de la exposición a alta temperatura y las tensiones inducidas por las fluctuaciones térmicas, pueden producir incluso el fallo del componente, debido a los diferentes coeficientes de expansión térmica entre el sustrato y la capa de productos de corrosión (Yoon y Jeong, 1999; Xie et al., 2006). Bajo estas condiciones, las tensiones generadas son capaces de propagar grietas en la capa formada e incluso pueden producir la delaminación de la película de productos oxidados, desprotegiendo así al material frente a los fenómenos de oxidación directa (Buscail et al., 2003; Yoshinaga et al., 2011).

El material 2.25Cr-1Mo se usa ampliamente en la fabricación de componentes no presurizados de la centrales térmicas (Muddle y Singh Raman, 2002). Por tanto, el objetivo de este trabajo es evaluar los efectos del ciclado térmico, comparándolos con condiciones isotermas a $550{ }^{\circ} \mathrm{C}$ y $650{ }^{\circ} \mathrm{C}$ durante 360 horas en aire.

\section{PROCEDIMIENTO EXPERIMENTAL}

\subsection{Material de partida}

El acero $2.25 \mathrm{Cr}-1 \mathrm{Mo}$ posee un tratamiento térmico previo de templado desde $940{ }^{\circ} \mathrm{C}$ y posteriormente un tratamiento de 30 minutos a $740{ }^{\circ} \mathrm{C}$, enfriando libremente hasta temperatura ambiente. Se cortaron muestras de dimensiones $30 \times 30 \times 4 \mathrm{~mm}$. Posteriormente se les proporcionó un acabado superficial previo a los ensayos de corrosión mediante el desbaste con $\mathrm{SiC}$ de granulometría 120\#. A continuación las muestras se limpiaron con etanol en ultrasonidos y se secaron con aire.

\subsection{Ensayos de ciclo térmico e isotermo}

Se realizaron ensayos de ciclo térmico sobre las muestras en atmósfera de aire. Las temperaturas de operación máximas durante cada ciclo se situaron a $550{ }^{\circ} \mathrm{C}$ y $650{ }^{\circ} \mathrm{C}$, y el tiempo de exposición total en cada caso fue de 360 horas. Los ciclos térmicos para las dos temperaturas se muestran en la Figura 1.

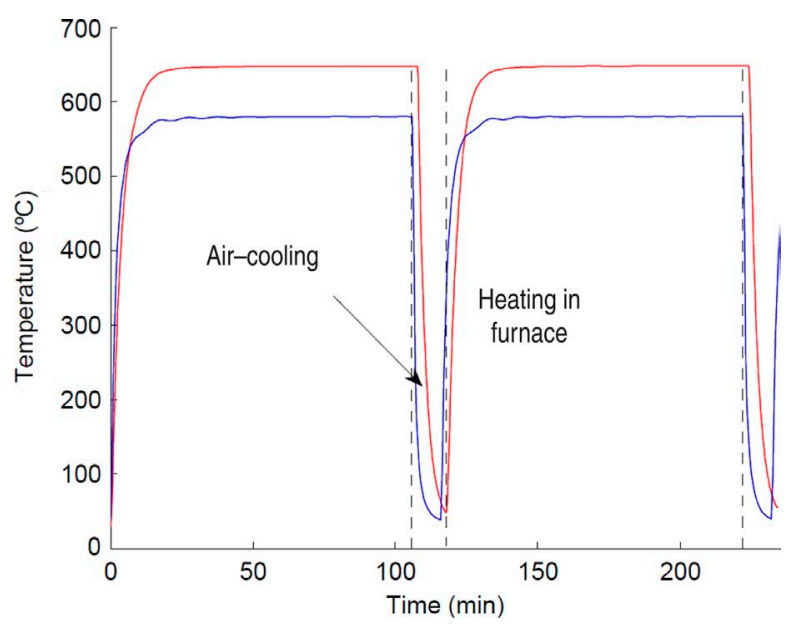

Figura 1. Ciclos térmicos a $550{ }^{\circ} \mathrm{C}$ y $650{ }^{\circ} \mathrm{C}$.

Los ensayos de ciclo térmico se realizaron en un horno de apertura de puerta controlada por un autómata, a través de un sistema neumático (Fig. 2). El sistema está formado por un horno y por una bandeja de posición variable que sustenta las muestras. El diseño del equipo permite el aislamiento térmico de la cámara del horno, cuando la bandeja se encuentra en el exterior, mediante una tapa cerámica que se acopla con el orificio de la puerta del horno. La salida de la bandeja activa un sistema de refrigeración forzada mediante aire. Las muestras están instrumentadas durante el proceso de choque térmico, gracias a transductores de tipo termopar conectados al sistema de adquisición de datos para verificar la temperatura de la muestra en cada momento. Se emplearon dos muestras para cada uno de los ensayos de oxidación cíclica $\left(550^{\circ} \mathrm{C}\right.$ y $\left.650{ }^{\circ} \mathrm{C}\right)$. Las pesadas se realizaron sobre cada una de las muestras que posteriormente se volvieron a introducir al sistema. Los tiempos de exposición en los que se realizó el estudio gravimétrico varían ligeramente de un ciclo a otro, dependiendo del instante en el que la muestra alcanza la temperatura ambiente. En ambos casos se realizaron al menos cinco medidas distribuidas durante las 360 horas de ensayo.

Los ensayos de oxidación isoterma se efectuaron a las mismas temperaturas, $550^{\circ} \mathrm{C}$ y $650^{\circ} \mathrm{C}$, con una rampa de calentamiento de $20^{\circ} \mathrm{C} \mathrm{min}-1$ y un posterior enfriamiento en el horno. Para realizar el estudio gravimétrico, se emplearon dos muestras para cada uno de los tiempos de exposición ensayados, que fueron 48, 120, 216 y 360 horas respectivamente.

\subsection{Difracción de Rayos X (DRX)}

La superficie de los materiales después del ensayo se estudió mediante DRX, para conocer los compuestos que aparecen en las capas más externas e identificar los óxidos formados mediante la identificación de la estructura cristalina. 
a)

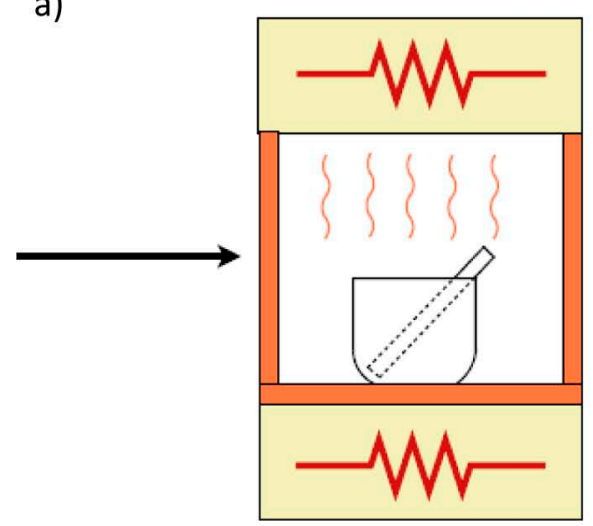

b)

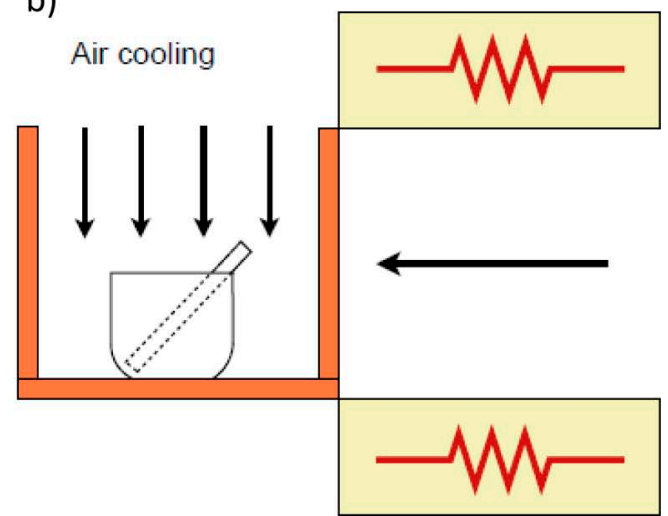

Figura 2. Esquema del horno de ciclo térmico: a) posición de calentamiento; b) posición de enfriamiento.

Tabla 1. Composición de la aleación 2.25Cr-1Mo obtenida mediante FRX (\% en peso)

\begin{tabular}{lccccccc}
\hline $\mathbf{C}$ & $\mathbf{S i}$ & $\mathbf{M n}$ & $\mathbf{C r}$ & $\mathbf{N i}$ & Al & Mo & Fe \\
\hline 0,15 & 0,43 & 0,57 & 2,23 & 0,23 & 0,08 & 0,96 & 95,35 \\
\hline
\end{tabular}

El equipo empleado para el estudio fue un difractómetro de polvo X'Pert MPD/MRD. El ánodo empleado es de cobre, que proporciona una radiación con una longitud de onda $\lambda_{\mathrm{Cu}}=1,54060 \times 10^{-10} \mathrm{~m}$. El intervalo de ángulos de Bragg empleado para cada ensayo fue entre $15 \mathrm{y}$ los $90^{\circ}$. El paso se estableció en $0,040^{\circ}$ y el tiempo de contaje fue 1 segundo. Posteriormente la indexación y resolución de las fases presentes en la zona de interacción, se realizó con la base de datos PDF4+.

\subsection{Espectroscopía Raman}

El equipo de medida empleado fue un espectrofotómetro Horiba Jobin-Yvon HR 800 UV con láser de Helio-Neon $(632,8 \mathrm{~nm})$. El intervalo de estudio estaba comprendido entre los 100 y $800 \mathrm{~cm}^{-1}$, con tiempos de acumulación de 15 segundos y dos adquisiciones por ciclo. Esta técnica fue usada tanto en las superficies oxidadas como en los cortes transversales.

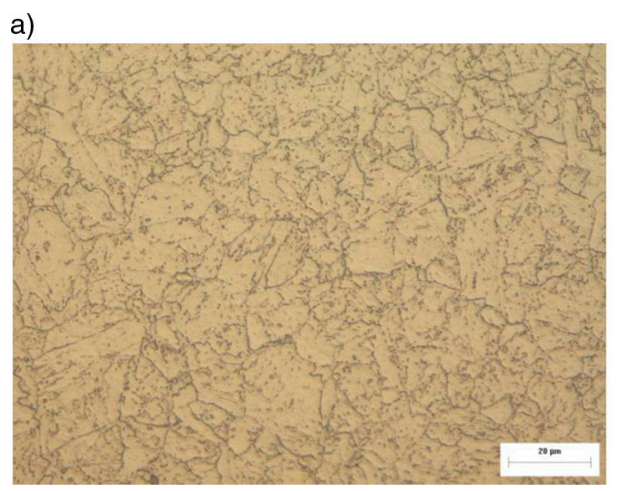

\subsection{Microscopía Óptica y Electrónica de Barrido (MEB)}

Para caracterizar la morfología de las películas de productos formados se emplearon técnicas de MEB. El equipo empleado fue un microscopio S-3400N de Hitachi, con detector de electrones secundarios, provisto de un sistema de microanálisis de Rayos X (EDX), para analizar semi-cuantitativamente la microestructura de las muestras analizadas. Además se utilizó un microscopio óptico de la marca Leica (DMR) equipado con cámara digital para la captura de imágenes.

\section{RESULTADOS Y DISCUSIÓN}

\subsection{Material de partida}

La composición media analizada mediante Fluorescencia de Rayos X (FRX) se muestra en la Tabla 1. En la Figura 3 se muestra la microestructura

b)

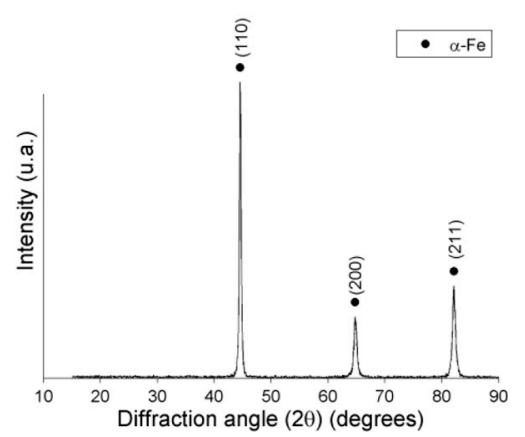

Figura 3. a) Micrografía óptica (500×) (atacado con Nital); b) difractograma de RX del material en estado de recepción. 
del acero tras la preparación metalográfica y el difractograma de RX realizado sobre el material en estado de recepción.

La microestructura del material está constituida por granos cristalinos de diferentes tamaños. Como se puede comprobar el microconstituyente principal de la aleación es la ferrita $(\alpha-\mathrm{Fe})$. Este hecho fue comprobado a través de la DRX en la que se identificó a esta misma fase cuya ficha es 04-014-0360.

\subsection{Ensayos a $550^{\circ} \mathrm{C}$}

En la Figura 4 se muestran las cinéticas de oxidación del material sometido a los ensayos isotermos y cíclicos.

Como queda patente en la figura, el efecto del ensayo de ciclo térmico sobre el material es más acusado que en el ensayo isotermo. Además, el material en condiciones isotérmicas se ajusta a una ley de tipo parabólica. Como está ampliamente aceptado, las tendencias parabólicas indican que los fenómenos difusivos en estado sólido son el proceso que controla la reacción, de acuerdo al modelo de Wagner (Chan et al. 2010; Wua et al., 2011).

Los valores de ganancia en masa por unidad de superficie expuesta son superiores en el ensayo cíclico con respecto a los obtenidos en el caso isotermo. Durante las primeras etapas de formación de la película del crecimiento, la cinética es parabólica y tras 120 horas de ensayo los valores experimentales se ajustan a una ley lineal. Por tanto, el proceso se describe mediante una ley cinética de tipo paralineal. En la Tabla 2 se muestran los resultados de los ajustes.

Después de los ensayos se empleó DRX para identificar los posibles productos de corrosión formados (Fig. 5).

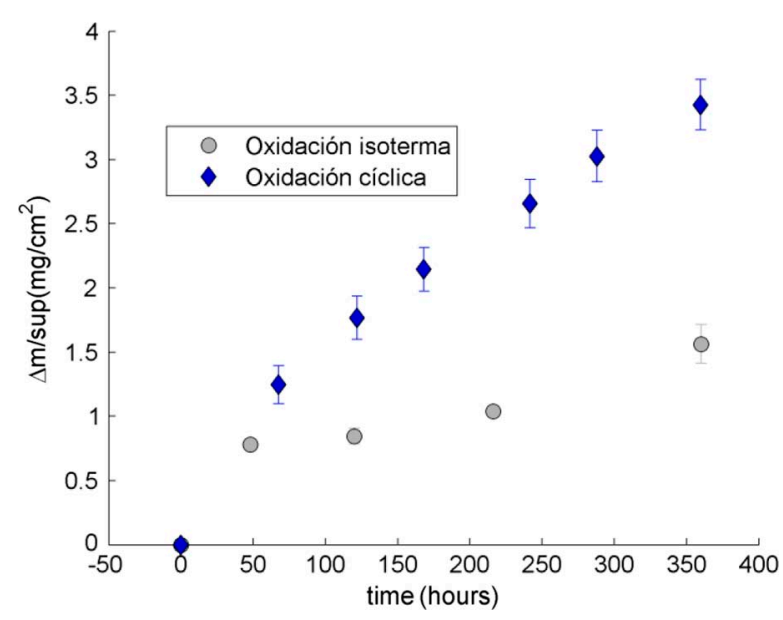

Figura 4. Comparación entre las cinéticas de oxidación isoterma y cíclica a $550{ }^{\circ} \mathrm{C}$.
TABla 2. Constantes de ajuste a las leyes cinéticas a $550{ }^{\circ} \mathrm{C}(2.25 \mathrm{Cr}-1 \mathrm{Mo})$

\begin{tabular}{lcc}
\hline Ensayo & $\mathbf{K}_{\mathrm{p}}$ & $\mathbf{R}^{2}$ \\
\hline Ciclo térmico & $(7,3 \pm 0,5) \cdot 10^{-3}$ & 0,99 \\
$\frac{\Delta m}{\text { sup }}=k \cdot t$ & $\left(\mathrm{mg} \mathrm{cm} \mathrm{h}^{-1}\right)$ & \\
Oxidación isoterma & $(1,9 \pm 0,7) \cdot 10^{-2}$ & \\
$\frac{\Delta m^{2}}{\text { sup }}=k_{p} \cdot t$ & $\left(\mathrm{mg}^{2} \mathrm{~cm}^{4} \mathrm{~h}^{-1}\right)$ & 0,94 \\
\hline
\end{tabular}

Para el ensayo cíclico se puede comprobar la presencia de dos fases oxidadas. Una estructura romboédrica cuyo grupo espacial es R-3c (167) de tipo corindón y una de tipo espinela, con grupo Fd-3m (227). Como es propio de este tipo de aceros, las fases son $\mathrm{Fe}_{2} \mathrm{O}_{3}$ (ficha 00-001-1053) y $\mathrm{Fe}_{3} \mathrm{O}_{4}$ (ficha 01-074-1909). En el caso de la oxidación isoterma (Fig. 5b) aparecen los mismos productos de corrosión.

En ambos casos es posible observar en los difractogramas las reflexiones del substrato, lo que indica el bajo espesor de la capa de productos oxidados. Asimismo en ambos casos la reflexión de mayor intensidad la produce la fase $\mathrm{Fe}_{2} \mathrm{O}_{3}$.

Debido a que los productos de corrosión son los mismos bajo las condiciones de ensayo analizadas, es necesario evaluar la morfología de las películas de productos oxidados.

Para caracterizar la superficie de los materiales, se empleó un microscopio electrónico de barrido. En las micrografías de la Figura 6, se muestra la superficie y la sección transversal del material sometido a condiciones de ciclo térmico a $550{ }^{\circ} \mathrm{C}$.

En la superficie de la muestra sin recubrimiento (Fig. 6a) sometida a condiciones de choque térmico durante 360 horas, se puede comprobar el modo en el que se ha producido la rotura continuada de las sucesivas películas de corrosión generadas durante el proceso, produciendo la desprotección del material. El corte transversal (Fig. 6b) muestra una capa de productos oxidados estratificada y con elevado número de defectos, que favorecen el proceso corrosivo. Este tipo de morfología, favorece el incremento de las tasas de pérdida de material y justifica la ley cinética lineal, a partir de la rotura de una capa de productos de corrosión, exenta de naturaleza protectora.

En las Figura 6c y $6 \mathrm{~d}$ se muestran los espectros Raman de dos zonas del material, la zona I correspondiente a las capas interiores y la zona II, o zona externa (Tabla 3). La zona interna sólo posee una fase con estructura espinela identificada como $\mathrm{Fe}_{3} \mathrm{O}_{4}$. En la zona externa aparece la fase $\mathrm{Fe}_{2} \mathrm{O}_{3}$ en la banda principal y el pico de espinela de $\mathrm{Fe}_{3} \mathrm{O}_{4}$. 
a)

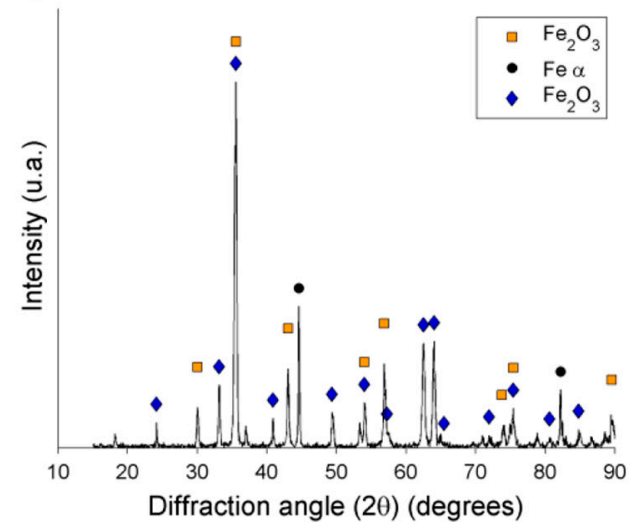

b)

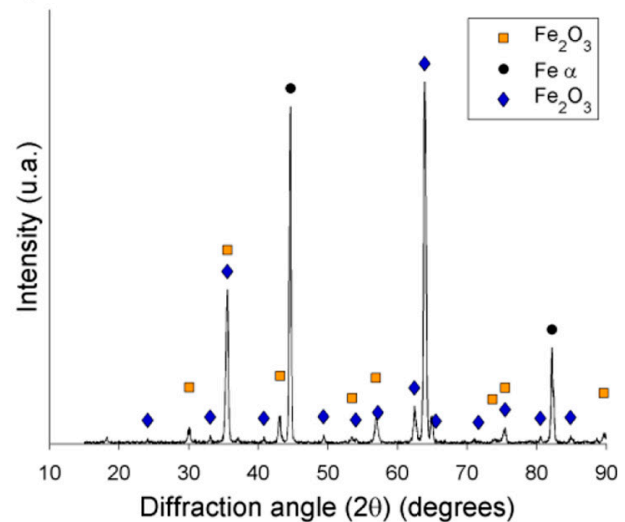

Figura 5. DRX de la muestra sometida a condiciones a $550^{\circ} \mathrm{C}$ después de 360 horas: a) oxidación cíclica; b) oxidación isoterma.
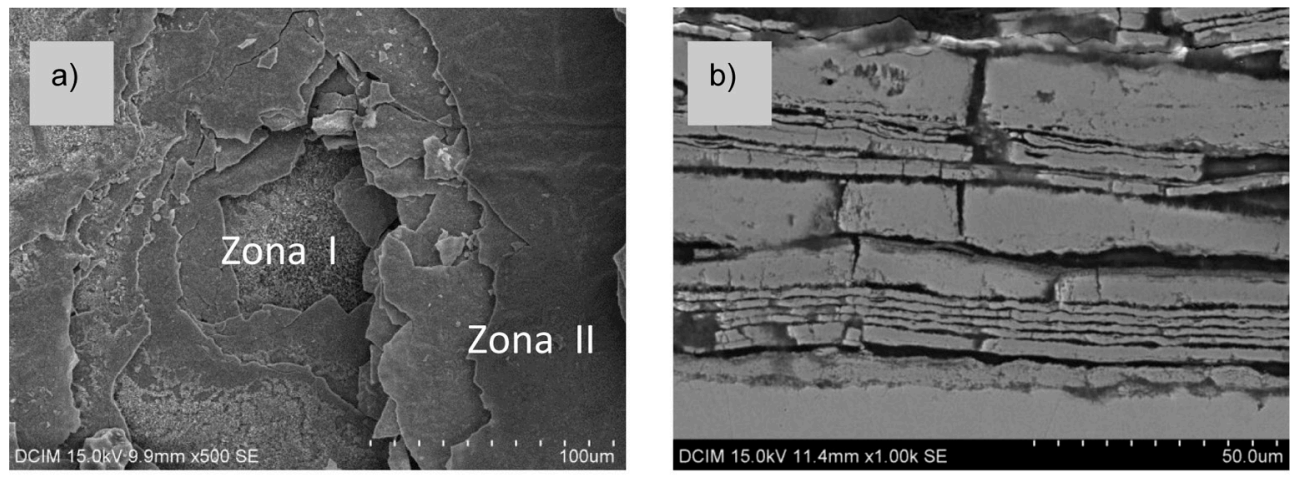

c)
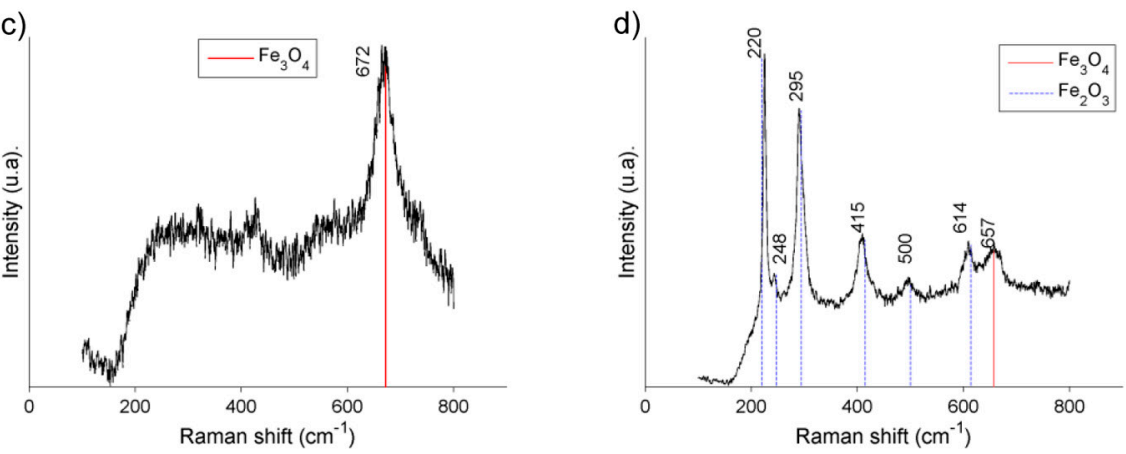

Figura 6. Micrografías MEB de la muestra sometida a ciclo térmico a $550^{\circ} \mathrm{C}$ : a) vista superficial (izquierda); b) sección transversal (derecha). Espectros Raman de: c) la zona I y d) la zona II.

TABLa 3. Posiciones Raman identificadas y referencias bibliográficas

\begin{tabular}{|c|c|c|c|c|c|}
\hline \multicolumn{3}{|c|}{$\mathrm{Fe}_{2} \mathrm{O}_{3}\left(\mathrm{~cm}^{-1}\right)$} & \multicolumn{3}{|c|}{$\mathrm{Fe}_{3} \mathrm{O}_{4}\left(\mathrm{~cm}^{-1}\right)$} \\
\hline (Gardiner et al., 1987) & (Seifert et al., 2010) & Experimental & (Hanesch, 2009) & (Seifert et al., 2010) & Experimental \\
\hline 220 & 226 & 220 & - & - & - \\
\hline 238 & 245 & 240 & - & - & - \\
\hline 288 & 292 & 287 & - & - & - \\
\hline 408 & 411 & 405 & - & - & - \\
\hline 498 & 497 & 489 & - & 532 & 539 \\
\hline 608 & 612 & 608 & 662 & 667 & 661 \\
\hline
\end{tabular}



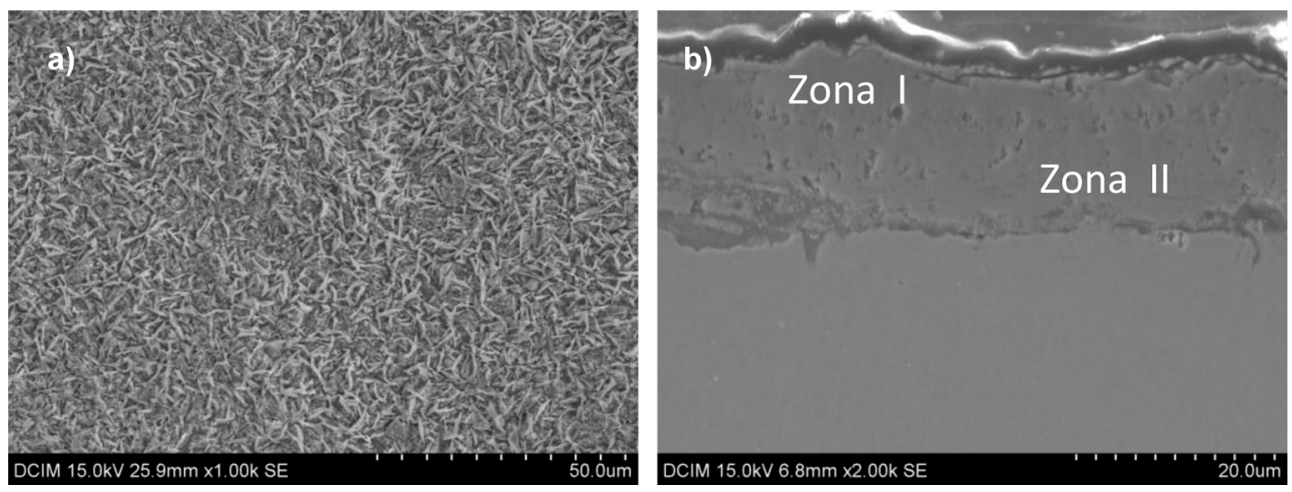

Figura 7. Micrografía MEB de la muestra sometida a oxidación isoterma a $550{ }^{\circ} \mathrm{C}$ durante 360 horas: a) vista superficial; b) sección transversal.

En la zona interna aparecen los picos de vibración correspondientes a la estructura cristalina $\mathrm{Fe}_{3} \mathrm{O}_{4}$ En el caso de la zona externa aparecen los picos de vibración identificados como la fase $\alpha \mathrm{Fe}_{2} \mathrm{O}_{3}$ además de picos atribuidos a $\mathrm{Fe}_{3} \mathrm{O}_{4}$.

La imagen general de la superficie (Fig. 7a) muestra la morfología de ataque del proceso corrosivo. Se trata de un avance generalizado sobre toda la superficie del material. Dicha capa se muestra libre de defectos, por lo que el material es capaz de generar una película protectora que le aísla de los fenómenos de corrosión a elevada temperatura. En la muestra de la sección transversal (Fig. 7b) se observan dos zonas. La zona más interna formada por óxidos de hierro y de cromo y la zona II por óxidos de hierro.

En la Figura 8 se muestran los análisis por EDX, que indican que la capa interna contiene óxidos ricos en hierro y cromo. La zona externa (zona II) es rica en hierro y oxígeno y se corresponde con la fase $\mathrm{Fe}_{2} \mathrm{O}_{3}$ y la zona interior contiene además de estos elementos, el cromo. Esta zona está formada fundamentalmente por $\mathrm{Fe}_{3} \mathrm{O}_{4}$, con algo de contenido en cromo que llega a la capa de óxido debido a los proceso de difusión.

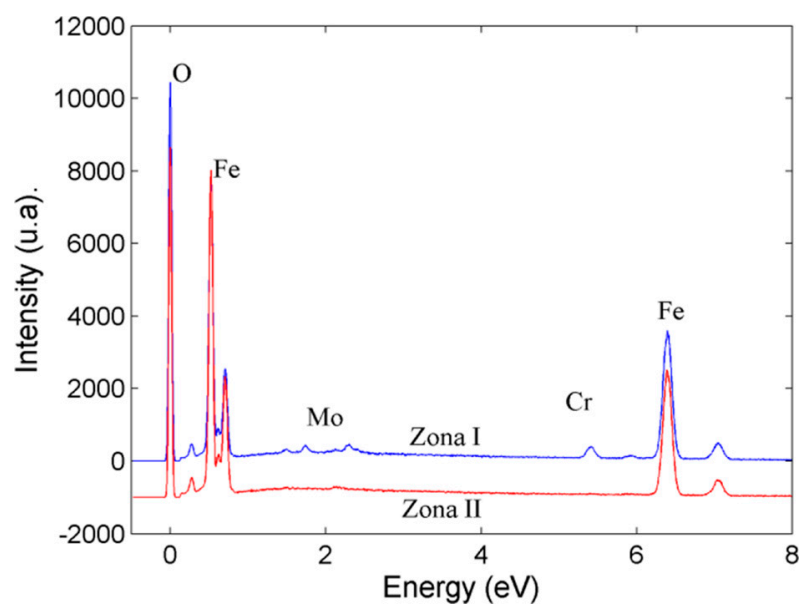

FIgURA 8. Análisis por EDX en la zona I y II de la muestra tratada a $550{ }^{\circ} \mathrm{C}$ durante 360 horas.

\subsection{Ensayos a $650{ }^{\circ} \mathrm{C}$}

En la Figura 9 se muestran las cinéticas de oxidación del acero $2.25 \mathrm{Cr}-1 \mathrm{Mo}$ en condiciones de oxidación isoterma y cíclica.

Como queda patente en las cinéticas de la figura, el efecto de la degradación en condiciones isotermas es más acusado que en el caso de las cíclicas. En la Tabla 4 se muestran las constantes de ajuste mediante el método de mínimos cuadrados.

Los valores de la constante parabólica son similares, por lo que el proceso de degradación tiene un efecto en el material comparable bajo ambas solicitaciones químicas y mecánicas. Los valores de las constantes cinéticas obtenidas en los casos de la oxidación isoterma y cíclica a $650{ }^{\circ} \mathrm{C}$, son superiores en más de dos órdenes de magnitud con respecto a los mismos ensayos realizados a $550{ }^{\circ} \mathrm{C}$. Este hecho indica que los procesos de degradación se ven claramente intensificados al aumentar $100{ }^{\circ} \mathrm{C}$ la temperatura de operación. Para poder estudiar las diferencias se realizó la caracterización de las muestras tras los correspondientes ensayos.

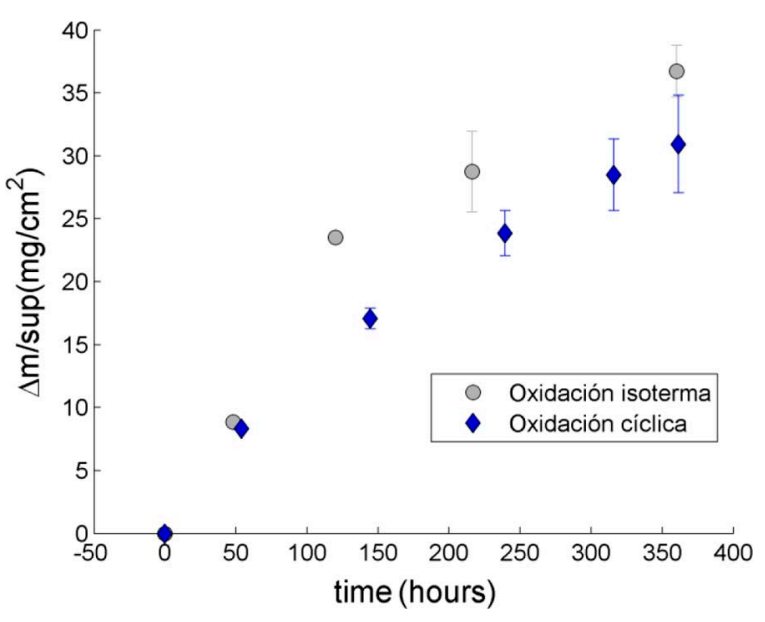

Figura 9. Comparación entre las cinéticas de oxidación isoterma y cíclica a $650^{\circ} \mathrm{C}$. 
TABLA 4. Constantes de ajuste a las leyes cinéticas a $650{ }^{\circ} \mathrm{C}$ $(2.25 \mathrm{Cr}-1 \mathrm{Mo})$

\begin{tabular}{lcc}
\hline Ensayo & $\mathbf{K}_{\mathrm{p}}$ & $\mathbf{R}^{2}$ \\
\hline Ciclo térmico & $\begin{array}{c}2,0 \pm 0,1 \\
\left(\mathrm{mg} \mathrm{cm}^{2} \mathrm{~h}^{-1}\right)\end{array}$ & 0,99 \\
$\left(\frac{\Delta m}{\text { sup }}\right)^{2}=k_{p} \cdot t$ & & \\
Oxidación isoterma & $\begin{array}{c}2,1 \pm 0,2 \\
\left(\mathrm{mg}^{2} \mathrm{~cm}^{4} \mathrm{~h}^{-1}\right)\end{array}$ & 0,94 \\
$\left(\frac{\Delta m}{\text { sup }}\right)^{2}=k_{p} \cdot t$ & &
\end{tabular}

En la Figura 10a se presenta el diagrama DRX de la muestra sometida a condiciones de ciclo térmico a $650{ }^{\circ} \mathrm{C}$. Como producto de corrosión aparece la $\mathrm{Fe}_{2} \mathrm{O}_{3}$ fundamentalmente y alguna reflexión del substrato. La presencia del substrato es debida a la rotura de la película durante el ciclado térmico. El difractograma del material sometido a oxidación isoterma se muestra en la Figura 10b. De nuevo, aparece un único producto de corrosión, con un modo de crecimiento en torno a una orientación preferente: se trata de la fase $\mathrm{Fe}_{2} \mathrm{O}_{3}$. La morfología de la película de productos oxidados formada durante la oxidación cíclica se muestra en la Figura 11.

En la imagen de MEB se puede comprobar que la capa más externa aparece de manera incompleta, debido a la delaminación parcial de la misma durante el proceso corrosivo o en la preparación metalográfica. De nuevo se puede contemplar la distribución estratificada de las películas de óxido debido a las tensiones cíclicas laterales, producidas durante el calentamiento y enfriamiento forzado. En la imagen de óptico se puede observar las tonalidades de los diferentes productos de corrosión formados. Asimismo, se propagan grietas en algunas capas y existen zonas que dan a entender que se ha producido un proceso de delaminación. En estas condiciones, el proceso corrosivo catastrófico produce tasas de reacción muy elevadas, generación de capas frágiles que no son capaces de soportar las tensiones térmicas y películas de productos oxidados carentes de carácter protector. Las muestras sometidas a oxidación isoterma a $650{ }^{\circ} \mathrm{C}$ se muestran en la Figura 12.

En la imagen superficial (Fig. 12a) se puede ver una superficie con un estado de oxidación muy
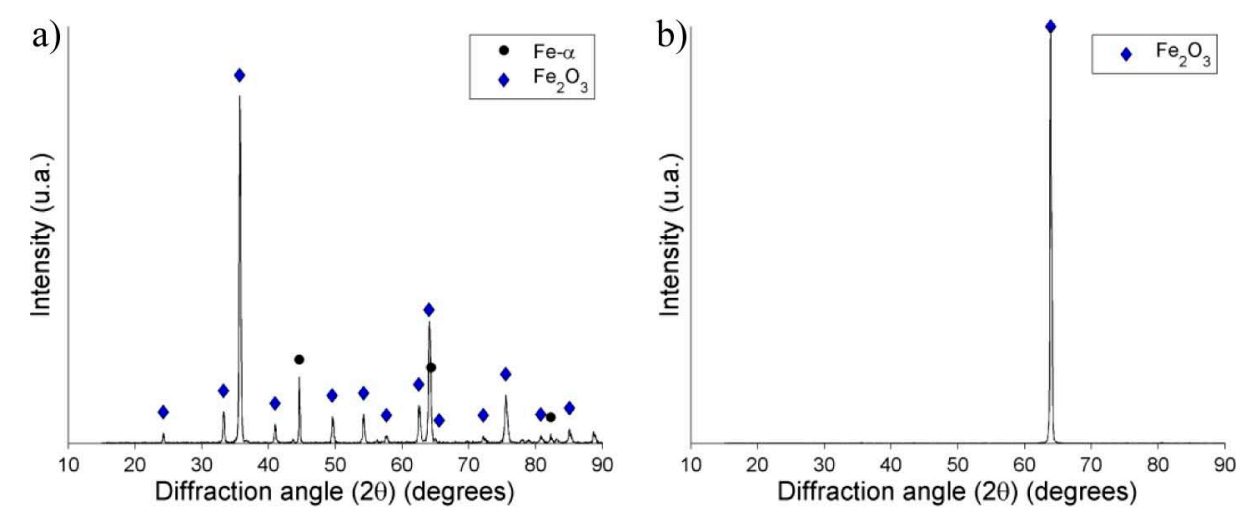

Figura 10. DRX de la muestra tratada a $650{ }^{\circ} \mathrm{C}$ durante 360 horas: a) ciclo térmico; b) oxidación isoterma.
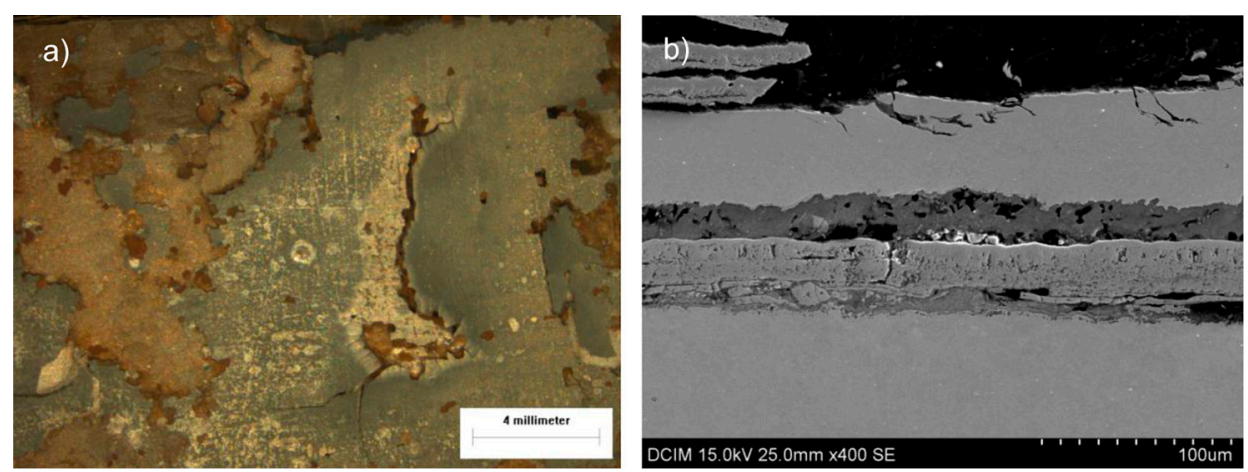

Figura 11. Muestra tratada en condiciones de oxidación cíclica a $650^{\circ} \mathrm{C}$ durante 360 horas: a) micrografía óptica de la vista superficial; b) micrografía MEB de la sección transversal. 


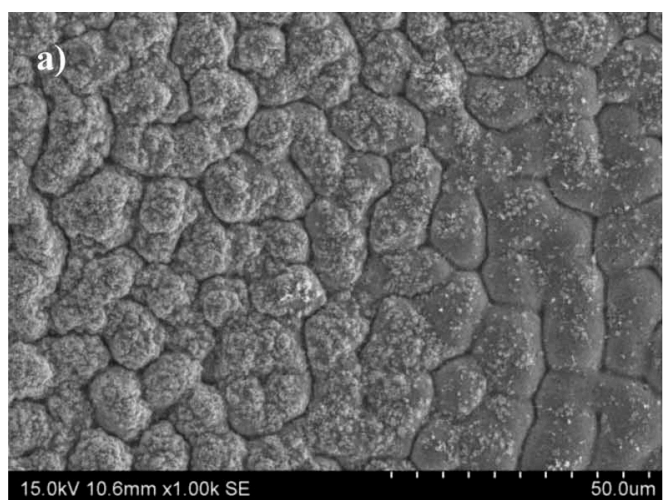

c)

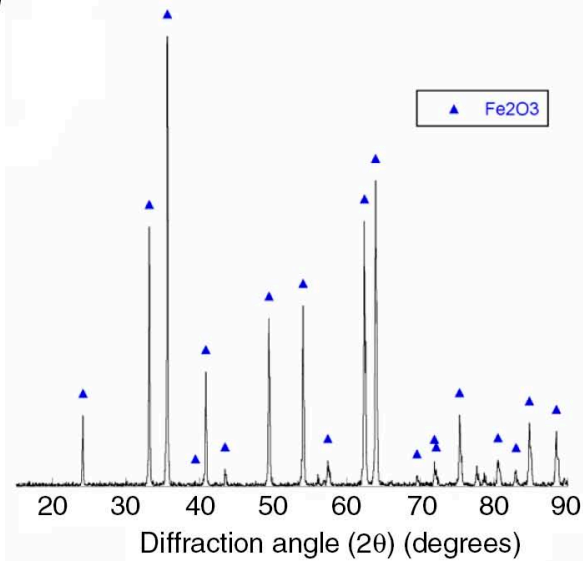

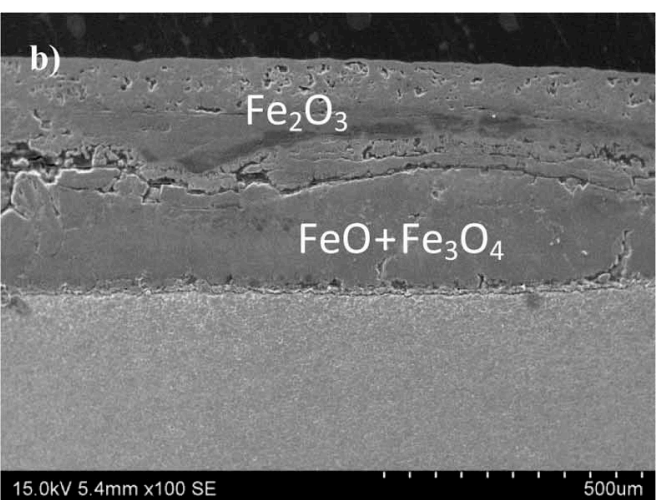

d)

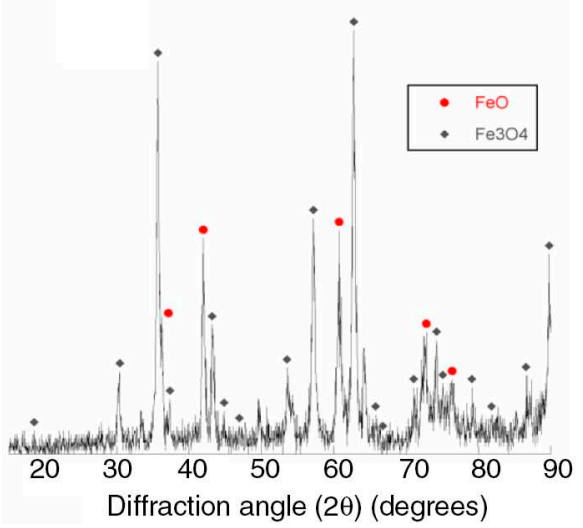

Figura 12. Micrografía MEB de la muestra sometida a oxidación isoterma durante 360 horas a $650^{\circ} \mathrm{C}$ : a) vista superficial (izquierda); b) sección transversal (derecha); c) difractograma de polvo de la capa externa; d) difractograma de polvo de la capa interna.

avanzado, donde los óxidos de hierro $\mathrm{Fe}_{2} \mathrm{O}_{3}$ crecen en forma de islas. Este tipo de mecanismos favorece la aparición de porosidad en la zona externa e incrementa la superficie de contacto entre el material y la película de productos oxidados.

En la micrografía de sección transversal (Fig. 12b) se puede comprobar que el espesor de la capa de óxido es de aproximadamente $400 \mu \mathrm{m}$, lo que indica que el material sufre una elevada pérdida de en estas condiciones de ensayo. Existen varias zonas diferenciadas; por un lado la capa interna presenta una elevada homogeneidad y ausencia de defectos, separada de la capa externa por una intercara discontinua que presenta una elevada concentración de defectos.

Mediante métodos mecánicos se extrajo polvo de la capa externa y de la interna y se analizó mediante DRX. La zona externa está formada por $\mathrm{Fe}_{2} \mathrm{O}_{3}$ y la interna posee dos fases, la magnetita $\left(\mathrm{Fe}_{3} \mathrm{O}_{4}\right)$ y la wustita $(\mathrm{FeO})$. Esta fase, es estable a partir de $570{ }^{\circ} \mathrm{C}$, posee una elevada falta de estequiometría y suele favorecer notablemente la difusión de especies. $\mathrm{Su}$ presencia en la película, por tanto, justifica las elevadas tasas de degradación a $650^{\circ} \mathrm{C}$ en las condiciones de oxidación isoterma.

\section{CONCLUSIONES}

El acero $2.25 \mathrm{Cr}-1 \mathrm{Mo}$ es capaz de operar a $550{ }^{\circ} \mathrm{C}$ en condiciones isotermas, produciendo valores de degradación moderados y una película de productos oxidados de bajo espesor.

En condiciones de oxidación cíclica a $550{ }^{\circ} \mathrm{C}$, las capas generadas se fracturan de manera consecutiva debido a las fluctuaciones térmicas inducidas durante el proceso. Este hecho produce rutas de fácil acceso para el oxígeno y favorecen el proceso de degradación del material.

En el caso de la oxidación isoterma a $650{ }^{\circ} \mathrm{C}$, se producen capas porosas y de gran espesor. La presencia del óxido $\mathrm{FeO}$ en el interior de la película favorece la difusión de las especies y justifica las elevadas tasas de degradación que sufre el material.

A $650{ }^{\circ} \mathrm{C}$ en condiciones de oxidación cíclica, se producen capas de óxido fracturadas y no adheridas a la superficie el material.

La diferencia de la constante cinética se incrementa en dos órdenes de magnitud en el ensayo realizado a $650{ }^{\circ} \mathrm{C}$ con respecto al de $550{ }^{\circ} \mathrm{C}$. 


\section{AGRADECIMIENTOS}

Los autores agradecen al proyecto de Comunidad de Madrid (ESTRUMAT S2009/MAT-1585) la financiación y el apoyo prestado para la realización de este trabajo.

\section{REFERENCIAS}

Buscail, H., Caudron, E., Cueff, R., Issartel, C., Perrier, S. y Riffard, F. 2003. Yttrium sol-gel coating effects on the cyclic oxidation behaviour of 304 stainless steel. Corros. Sci. 45 (12), 2867-2880. http://dx.doi.org/10.1016/ S0010-938X(03)00114-8

Chen, R.Y., Yuen, W.Y.D. 2010. Short-time Oxidation Behavior of Low-carbon, Low-silicon Steel in Air at 850$1,180{ }^{\circ} \mathrm{C}-\mathrm{III}:$ Mixed Linear-and-Parabolic to Parabolic Transition Determined Using Local Mass-Transport Theories. Oxid. Met. 74 (5-6), 353-373. http://dx.doi. org/10.1007/s11085-010-9212-8.

Gardiner, D.J., Littelton, C.J. y Thomas, K.M. 1987. Distribution and characterization of high temperature air corrosion products on iron-chromium alloys by Raman microscopy. Oxid. Met. 27 (1-2), 57-72. http://dx.doi.org/10.1007/ BF00656729.

Hanesch, M. 2009. Raman spectroscopy of iron oxides and (oxy) hidroxides at low laser power and possible applications in environmetal magnetic studies. Geophys. J. Int. 177 (3), 941948. http://dx.doi.org/10.111//j.1365-246X.2009.04122.x.

Henry, J.F., Tanzosh, J., Stanko, G., Shingledecker, J., Vitalis, B., Purgert, R. y Viswanathan, R. 2005. U.S. program on materials technology for ultra-supercritical coal power plants. J. Mater. Eng. Perform. 14 (3), 281-292. http:// dx.doi.org/10.1361/10599490524039.
Muddle, B.C. y Singh Raman, R.K. 2002. High temperature oxidation in the context of life assessment and microstructural degradation of weldments of $2.25 \mathrm{Cr}-1 \mathrm{Mos}$ steel. Int. J. Pres. Ves. Pip. 79 (8-10), 585-590. http://dx.doi. org/10.1016/S0308-0161(02)00093-5.

Sarver, J., Tanzosh , J.M. y Viswanathan, R. 2006. Boiler materials for ultra-supercritical coal power plants-Steamside oxidation. J. Mater. Eng. Perform. 15 (3), 255-274. http:// dx.doi.org/10.1361/105994906X108756

Seifert, W., Thomas, R. y Rhede, D.F. 2010. Origin of coexisting wustite, $\mathrm{MgFe}$ and REE phosphate minerals in graphite-bearing fluorapatite from the Rumburk granite. Eur. J. Mineral. 22 (4), 495-507. http://dx.doi. org/10.1127/0935-1221/2010/0022-2034.

Viswanathan, R. y Bakker, W. 2001. Materials for ultrasupercritical coal power plants-Boiler materials: Part 1. J. Mater. Eng. Perform. 10 (1), 81-95. http://dx.doi. org/10.1361/105994901770345394.

Wu, X., Kuang, W., Han, E.H., Rao, J. 2011. The mechanism of oxide film formation on Alloy 690 in oxygenated high temperature water, Corros. Sci. 53 (11), 3853-3860. http:// dx.doi.org/10.1016/j.corsci.2011.07.038.

Xie Y., Wang M., Zhang G. y Chang M. 2006. Analysis of superalloy turbine blade tip cracking during service. Eng. Fail. Anal. 13 (8), 1429-1436. http://dx.doi.org/10.1016/j. engfailanal.2005.07.022

Yoon, K.B. y Jeong, D.G. 1999. Oxidation failure of radiant heater tubes. Eng. Fail. Anal. 6 (2), 101-112. http://dx.doi. org/10.1016/S1350-6307(98)00033-8.

Yoshinaga M., Kishimoto H., Yamaji K., Brito M.E., Xiong Y., Yokokawaza H. y Horita T. 2011. Effects of Thermal Cycling on the Formation of Oxide Scale of $\mathrm{Fe}-\mathrm{Cr}$ Alloy Interconnects for Solid Oxide Fuel Cells. Int. J. Appl. Ceram. Technol. 8 (6), 1374-1381. http://dx.doi. org/10.1111/j.1744-7402.2010.02600.x.

Young, D.J. 2008. High Temperature oxidation an corrosion of metals, Ed. Elsevier, Amsterdam, Holanda, pp. 497-498. 